\title{
On the strong unique continuation property of a degenerate elliptic operator with Hardy-type potential
}

\author{
Agnid Banerjee ${ }^{1} \cdot$ Arka Mallick $^{2}$
}

Received: 22 July 2018 / Accepted: 3 May 2019 / Published online: 17 May 2019

(c) Fondazione Annali di Matematica Pura ed Applicata and Springer-Verlag GmbH Germany, part of Springer Nature 2019

\section{Abstract}

In this paper, we prove the strong unique continuation property for the following degenerate elliptic equation

$$
\Delta_{z} u+|z|^{2} \partial_{t}^{2} u=V u, \quad(z, t) \in \mathbb{R}^{N} \times \mathbb{R}
$$

where the potential $V$ satisfies either of the following growth assumptions

$$
\begin{aligned}
& |V(z, t)| \leq \frac{f(\rho(z, t))}{\rho(z, t)^{2}} \text {, where } \rho \text { is as in (2.1) and } \\
& \quad f \text { satisfies the Dini integrability condition as in (1.3). }
\end{aligned}
$$

or when

$$
|V(z, t)| \leq C \frac{\psi(z, t)^{\epsilon}}{\rho(z, t)^{2}}, \text { for some } \epsilon>0 \text { with } \psi \text { as in (2.6) and } N \text { even. }
$$

This extends some of the previous results obtained in [18] for this subfamily of BaouendiGrushin operators. As corollaries, we obtain new unique continuation properties for solutions $u$ to

$$
\Delta_{\mathbb{H}} u=V u
$$

with certain symmetries as expressed in (1.6) where $\Delta_{\mathbb{H}}$ corresponds to the sub-Laplacian on the Heisenberg group $\mathbb{H}^{n}$.

Keywords Unique continuation - Baouendi-Grushin operator · Hardy-type potential · Carleman estimates

First author is supported in part by SERB Matrix Grant MTR/2018/000267.

$\bowtie \quad$ Agnid Banerjee

agnid@math.tifrbng.res.in

Arka Mallick

arka.mallick@epfl.ch; arkamallick02@gmail.com

1 TIFR Centre for Applicable Mathematics, Post Bag No. 6503, Sharadanagar, Bangalore 560065, India

2 Department of Mathematics, EPFL SB CAMA, Station 8, 1015 Lausanne, Switzerland 
Mathematics Subject Classification $35 \mathrm{~J} 70 \cdot 35 \mathrm{~J} 75$

\section{Introduction}

An operator $L$ (local or non-local) is said to possess the strong unique continuation property in the $L^{p}$ sense if any non-trivial solution $u$ to

$$
L u=0
$$

in a (connected) domain $\Omega \subset \mathbb{R}^{n}$ cannot vanish to infinite order in the $L^{p}$ mean at any point in $\Omega$. We refer to Definition 4.1 for the precise notion of vanishing to infinite order in the $L^{p}$ mean.

The fundamental role played by strong unique continuation theorems in the theory of partial differential equations is well known. We recall that in his foundational paper in 1939 (see [8]), T. Carleman established the strong unique continuation property for

$$
\Delta u=V u
$$

in $\mathbb{R}^{2}$ under the assumption that $V$ is in $L^{\infty}$. This result was subsequently extended by several mathematicians to arbitrary dimension and also to equations with variable coefficients. In this direction, we refer to the pioneering work of Aronszajn-Krzywicki-Szarski (see [2]), where strong unique continuation property for elliptic operators with Lipschitz principal part was established by using generalization of the estimates of Carleman. About twenty years later, a different geometric approach independent of the Carleman estimates came up in the seminal works of Garofalo-Lin in 1986, see [19,20]. Their method, which is based on the almost monotonicity of a generalized frequency function, allowed them to obtain new quantitative information on the zero set of solutions to divergence form elliptic equations and, in particular, encompassed the results of [2]. The reader should note that such a frequency function approach has its roots in the well-known work of Almgren [3] which plays a crucial role in the regularity theory of mass minimizing currents.

There has also been an interest of mathematicians working in partial differential equations and mathematical physics to focus on the unique continuation property for equations with unbounded lower-order terms. Subsequent developments in this direction have culminated with Jerison and Kenig's celebrated result on the strong unique continuation property for

$$
\Delta u=V u
$$

with $V \in L_{l o c}^{n / 2}\left(\mathbb{R}^{n}\right)$, see [28]. Their paper has inspired much progress in the subject, and nowadays, the picture for second-order uniformly elliptic equations is almost complete. See, for instance, [30] where analogous results have been established for variable coefficient operators with Lipschitz principal part. We also refer to the work of Pan [31], where strong unique continuation property for elliptic equations with scaling critical Hardy-type potentials has been established, as well as to that of Chanillo-Sawyer (see [9]) for strong unique continuation results with potentials in the Fefferman-Phong class.

On the contrary, not so well understood is the situation concerning sub-elliptic operators. It turns out that unique continuation is generically not true in such context. This follows from a counterexample due to Bahouri [7], where the author showed that unique continuation is not true for even smooth and compactly supported perturbations of the sub-Laplacian on the Heisenberg group $\mathbb{H}^{n}$. The first positive result in this direction came up in the work of Garofalo-Lanconelli [21] where among other important results, the authors showed that 
strong unique continuation result holds for solutions $u$ to

$$
\Delta_{\mathbb{H}} u=V u
$$

when $u$ has certain symmetries as expressed in (1.6) and with certain growth conditions on $V$, see Theorem 5.1. Here, $\Delta_{\mathbb{H}}$ denotes the standard sub-Laplacian on $\mathbb{H}^{n}$. We also refer to the papers [4,24] for the extension of the unique continuation result in [21] to Carnot groups of arbitrary step with appropriate symmetry assumptions on the solution $u$ which can be thought of as analogous to that in (1.6). It is to be noted that the results in $[4,21,24]$ follow the circle of ideas as in the fundamental works $[19,20]$ based on Almgren-type frequency function approach.

In a somewhat related direction, the study of strong unique continuation for zero-order perturbations of the following degenerate Baouendi-Grushin-type operators

$$
\mathcal{B}_{\beta} u=\Delta_{z} u+\frac{|z|^{2 \beta}}{4} \Delta_{t} u, \quad z \in \mathbb{R}^{N}, t \in \mathbb{R}^{m}, \beta>0
$$

was initiated by Garofalo in [18], where the author introduced an Almgren-type frequency function associated with $\mathcal{B}_{\beta}$ and proved that such a frequency is bounded for solutions $u$ to

$$
\mathcal{B}_{\beta} u=V u
$$

when $V$ satisfies the following growth assumption

$$
|V(z, t)| \leq C \frac{f(\rho(z, t))}{\rho(z, t)^{2}} \psi(z, t),
$$

for some non-decreasing $f$ which is Dini-integrable, i.e.

$$
\int_{0}^{R_{0}} \frac{f(r)}{r}<\infty, \text { for some } R_{0}>0
$$

and where

$$
\rho(z, t)=\left(|z|^{2(\beta+1)}+(\beta+1)^{2}|t|^{2}\right)^{\frac{1}{2(\beta+1)}} \quad \text { and } \quad \psi(z, t)=\frac{|z|^{2 \beta}}{\rho(z, t)^{2 \beta}},
$$

Consequently, using the boundedness of such a frequency, the author inferred that the $L^{2}$ doubling property of solutions to (1.1) follows which in particular implies the strong unique continuation property. Also the case when $V=V^{+}-V^{-}$satisfies

$$
\begin{aligned}
& 0<V^{+}(z, t) \leq C \frac{\psi}{\rho(z, t)^{2}}, \\
& 0 \leq V^{-}(z, t) \leq \delta \frac{\psi}{\rho(z, t)^{2}},
\end{aligned}
$$

for some $\delta>0$ small enough depending on $N, m$ and $\beta$ was studied in the very same paper and in which case a slightly weaker version of unique continuation property was established (see Theorem 4.4 in [18]). The results in [18] were subsequently extended in [4,26] to variable coefficient Baouendi-Grushin operators. Note that the weight $\psi$ in $(1.2) /(1.4)$ degenerates on the submanifold $\{z=0\}$ and so the result in [18] does not allow to take $V \in L^{\infty}$. The natural appearance of this degenerate weight $\psi$ can be seen from the fact that if

$$
u=f(\rho(z, t))
$$


then

$$
L u=\psi\left(f^{\prime \prime}(\rho)+\frac{Q-1}{\rho} f^{\prime}(\rho)\right), \quad \text { where } Q=N+(\beta+1) m .
$$

When $\beta=1, m=1$, we note that the operator $\mathcal{B}_{\beta}$ is intimately connected to the subLaplacian on $\mathbb{H}^{n}$. In order to see such a connection, we note that with respect to the standard coordinates $(z, t)=(x, y, t)$ on $\mathbb{H}^{n}\left(=\mathbb{R}^{n} \times \mathbb{R}^{n} \times \mathbb{R}\right)$, the sub-Laplacian is given by

$$
\Delta_{\mathbb{H}}=\Delta_{z}+\frac{|z|^{2}}{4} \partial_{t}^{2}+\sum_{i=1}^{n} \partial_{t}\left(y_{j} \partial_{x_{j}}-x_{j} \partial_{y_{j}}\right)
$$

Now if $u$ is a smooth function that annihilates the vector field $T=\sum_{i=1}^{n}\left(y_{j} \partial_{x_{j}}-x_{j} \partial_{y_{j}}\right)$ (i.e. $T u \equiv 0$ ), then (up to a normalization factor of 4 ) we have that

$$
\Delta_{\mathbb{H}} u=\mathcal{B}_{1} u, \quad \text { for } m=1 .
$$

Note that it is not difficult to recognize that

$$
T u=0 \text { iff } u\left(\mathrm{e}^{i \theta} z, t\right)=u(z, t) .
$$

Said differently, $T u=0$ if and only if $u$ is invariant with respect to the natural action of the torus on $\mathbb{H}^{n}$.

In this very same case (i.e. when $\beta=1$ and $m=1$ ), by establishing very delicate $L^{p}-L^{q}$ Carleman estimates as stated in (3.2), Garofalo and Shen in [25] obtained strong unique continuation for (0.1) when $V \in L_{l o c}^{r}\left(\mathbb{R}^{N+1}\right)$ for $r>N=Q-2$ when $N$ is even and $r>\frac{2 N^{2}}{N+1}$ when $N$ is odd and hence succeeded in removing the degenerate weight $\psi$ from their growth assumption unlike that in (1.2) or (1.4) for this subfamily of $\left\{\mathcal{B}_{\beta}\right\}$. The reader should note that the approach in [25] in turn is inspired by that of Jerison's work in [27] where a simpler proof of the Jerison-Kenig's Carleman inequality was discovered.

Now for a historical account, we recall that a more general class of operators modelled on $\mathcal{B}_{\beta}$ was first introduced by Baouendi who studied the Dirichlet problem in weighted Sobolev spaces (see [6]). Subsequently, Grushin in [22,23] studied the hypoellipticity of the operator $\mathcal{B}_{\beta}$ when $\beta \in \mathbb{N}$ and showed that this property is, however, affected by the addition of lower-order terms. We would also like to refer to the papers [12,13] for Hölder regularity of weak solutions to general equations modelled on $\mathcal{B}_{\beta}$. Remarkably, the operator of Baouendi also played an important role in the recent work [29] on the higher regularity of the free boundary in the classical Signorini problem. We would also like to mention that a version of the Almgren-type monotonicity formula for $\mathcal{B}_{\beta}$ played an extensive role in [11] on the obstacle problem for the fractional Laplacian.

Therefore, given the relevance of these sub-elliptic operators in various contexts, in this paper we study the strong unique continuation for zero-order perturbations of the operator $\mathcal{B}_{1}$ (when $m=1$ ) with singular potentials of Hardy type. We first show that quite interestingly, by employing the $L^{p}-L^{q}$ type Carleman estimate derived in [25], one can prove strong unique continuation property for $(0.1)$ (see Theorem 4.2) when the potential $V$ satisfies the following growth condition

$$
|V(z, t)| \leq \frac{f(\rho(z, t))}{\rho(z, t)^{2}} \quad \text { for a.e. }(z, t) \in B_{R_{0}} \text { and } f \text { satisfies (1.3), }
$$

i.e. we show that in the growth condition (1.2) as treated in [18], the degenerate weight $\psi$ can be removed from the growth assumption for this subfamily of operators for the validity of strong unique continuation. Typical representatives of $f$ satisfying (1.3) are $f(r)=C r^{\epsilon}$ 
$(\epsilon>0)$ or $f(r)=C|\log (1 / r)|^{-\alpha}(\alpha>1)$ and therefore the growth assumption in (1.7) can be thought of as an "almost" Hardy-type growth condition.

Then by using the $L^{2}$ estimates for projection operators $P_{k}$ as established in [25] [(see (2.11) for the definition of $P_{k}$ ], we establish a certain $L^{2}-L^{2}$-type Carleman estimate where we obtain a particular asymptotic behaviour of the constant involved in terms of a parameter $s$ which corresponds to the exponent of the singular weight in the Carleman inequality (see the estimate in Theorem 3.3). Using such an asymptotic behaviour of the constant, we adapt an argument in [31] to our sub-elliptic setting and consequently obtain an analogous strong unique continuation result for Eq. (0.1) when the potential $V$ satisfies the following Hardytype growth condition

$$
|V(z, t)| \leq C \frac{\psi(z, t)^{\epsilon}}{\rho(z, t)^{2}} \quad \text { for a.e. }(z, t) \in B_{R_{0}}, \epsilon>0 \text { and } N \text { even, }
$$

which again improves the growth assumption in (1.4) for this subclass of Baouendi-Grushin operators (see Theorem 4.3 and also Theorem 4.4 for the corresponding result when $N$ is odd). The reader should note that our result in Theorem 4.3 is new even for $\epsilon=1$ because the growth assumption in (1.4) requires $\delta$ to be sufficiently small. We would also like to mention that although we closely follow the approach of [31] in parts, it has nonetheless required some delicate modifications in our setting as the reader can see in the proof of Theorem 4.3 in Sect. 4. This is essentially due to the presence of the degenerate weight $\psi$ in our Carleman estimates. We also note that a generic potential $V$ satisfying our growth conditions in (1.7) or (1.8) need not be in $L^{r}$ for the range of $r$ dealt in [25], and therefore, the class of potentials covered in our work are somewhat complementary to that treated in [25].

A few open problems as well as remarks are listed in order:

(1) We note that in our growth condition (1.8), the parameter $\epsilon$ which corresponds to the exponent of $\psi$ can be taken arbitrarily small. Said differently, we show in our Theorem 4.3 that the degenerate weight $\psi$ can be "almost" removed in the Hardy-type growth assumption. It, however, remains to be seen whether in (1.8), one can get rid of the degenerate weight $\psi$ completely from the growth condition, i.e. whether one can take $\epsilon=0$ in (1.8) so that Theorem 4.3 continues to hold. This appears to be a challenging open problem to which we would like to come back in a future study.

(2) It also appears interesting to look at generalization of our unique continuation results as well as that of [25] for the case when $m>1$, i.e. for equations of the type

$$
\Delta_{z} u+|z|^{2} \Delta_{t} u=V u, \quad(z, t) \in \mathbb{R}^{N} \times \mathbb{R}^{m}
$$

The reader should note that this would consequently have similar applications to unique continuation properties for sub-Laplacian-type equations on $H$ - type groups (see Section 9 in [24] for a detailed account on this aspect). This seems to be a challenging issue as well where one would need to establish estimates similar to that stated in Theorem 3.1 for appropriate projection operators.

(3) It would also be interesting to look at backward uniqueness results for zero-order perturbations of the parabolic counterpart of the operators as in $(0.1)$ or more generally of the ones as in (1.9). We refer the reader to the interesting papers [15-17,32] for the corresponding results in the case of uniformly parabolic operators.

The paper is organized as follows: In Sect. 2, we introduce certain relevant notions and gather some known results. In Sect. 3, we establish a certain $L^{2}-L^{2}$-type Carleman estimate with a particular asymptotic behaviour of the corresponding constant as mentioned above using which we prove our strong unique continuation result Theorem 4.3. In Sect. 4, we 
prove our main results in Theorems 4.2 and 4.3 using the Carleman estimates in Sect. 3. Finally in Sect. 5, we show application of our results to a new unique continuation property for stationary Schrödinger equations on the Heisenberg group $\mathbb{H}^{n}$.

\section{Preliminaries}

The content of this section is essentially borrowed from [25]. The main goal is to introduce a suitable polar coordinates with respect to the non-isotropic gauge function defined in (2.1) below and show how the Grushin operator interacts with these newly introduced polar coordinates.

$$
\rho(z, t)=\left(|z|^{4}+4 t^{2}\right)^{\frac{1}{4}}, \quad z \in \mathbb{R}^{N}, t \in \mathbb{R} .
$$

For $0<\phi<\pi, 0<\theta_{i}<\pi, i=1,2, \ldots, N-2$ and $0<\theta_{N-1}<2 \pi$ let

$$
\left\{\begin{array}{l}
z_{1}=\rho \sin ^{\frac{1}{2}} \phi \sin \theta_{1} \ldots \sin \theta_{N-2} \sin \theta_{N-1} \\
z_{2}=\rho \sin ^{\frac{1}{2}} \phi \sin \theta_{1} \ldots \sin \theta_{N-2} \cos \theta_{N-1} \\
\vdots \\
z_{N}=\rho \sin ^{\frac{1}{2}} \phi \cos \theta_{1} \\
t=\frac{\rho^{2}}{2} \cos \phi
\end{array}\right.
$$

Then as computed in [25], we have

$$
\begin{aligned}
r & =|z|=\rho \sin ^{\frac{1}{2}} \phi, \\
\mathrm{d} z \mathrm{~d} t & =\frac{1}{2} \rho^{N+1}(\sin \phi)^{\frac{N-2}{2}} \mathrm{~d} \rho \mathrm{d} \phi \mathrm{d} \omega \\
\mathcal{L} & =\Delta_{z}+|z|^{2} \frac{\partial^{2}}{\partial t^{2}}=\sin \phi\left(\frac{\partial^{2}}{\partial \rho^{2}}+\frac{N+1}{\rho} \frac{\partial}{\partial \rho}+\frac{4}{\rho^{2}} \mathcal{L}_{\sigma}\right),
\end{aligned}
$$

where $d \omega$ denotes the surface measure on $S^{N-1}$ and

$$
\mathcal{L}_{\sigma}=\frac{\partial^{2}}{\partial \phi^{2}}+\frac{N}{2} \frac{\cos \phi}{\sin \phi} \frac{\partial}{\partial \phi}+\frac{1}{(2 \sin \phi)^{2}} \Delta_{S^{N-1}} .
$$

Here $\sigma=(\phi, \omega), \omega \in S^{N-1}$ and $\Delta_{S^{N-1}}$ denotes the Laplace-Beltrami operator on $S^{N-1}$. Notice that

$$
\sin \phi=\psi
$$

where

$$
\psi(z, t):=\frac{r^{2}}{\rho^{2}}=\frac{|z|^{2}}{\left(|z|^{4}+4 t^{2}\right)^{\frac{1}{2}}}
$$

The following lemma characterizes the spherical harmonics for the Grushin operator.

Lemma 2.1 Let $k$ be non-negative integer and $l=k(\bmod 2)$, with $0 \leq l \leq k$. Suppose that $Y_{l}$ is a spherical harmonic of degree $l$ for $\Delta_{S^{N-1}}$. Then

$$
g(\phi, \omega)=\sin ^{\frac{l}{2}} \phi C_{\frac{k-l}{2}}^{\frac{l}{2}+\frac{N}{4}}(\cos \phi) Y_{l}(\omega)
$$


satisfies $\mathcal{L}_{\sigma} g=-\frac{k(N+k)}{4} g$. Here, $C_{\frac{k-l}{2}}^{\frac{l}{2}+\frac{N}{4}}(\tau)$ is an ultraspherical (or Gegenbauer) polynomial. We refer to $p .174$ in [15] for relevant details.

As in [25], we now define,

$\mathcal{H}_{k}=\operatorname{span}\left\{\sin ^{\frac{l}{2}} \phi C_{\frac{k-l}{2}}^{\frac{l}{2}+\frac{N}{4}}(\cos \phi) Y_{l, j}(\omega) \mid j=1,2, \ldots, d_{l}, 0 \leq l \leq k, l=k(\bmod 2)\right\}$,

where $d_{l}=\frac{(N+2 l-2) \Gamma(N+l-2)}{\Gamma(l+1) \Gamma(N-1)}$ and $\left\{Y_{l, j}\right\}_{j=1, \ldots, d_{l}}$ denote an orthonormal basis for the space of spherical harmonics of degree $l$ on $S^{n-1}$. By taking $\rho=1$ in (2.2), we can parametrize

$$
\Omega=\left\{(z, t) \in \mathbb{R}^{N+1} \mid \rho(z, t)=\left(|z|^{4}+4 t^{2}\right)^{\frac{1}{4}}=1\right\}
$$

and consider the measure given by,

$$
\mathrm{d} \Omega=\sin ^{\frac{N}{2}} \mathrm{~d} \phi \mathrm{d} \omega .
$$

Then as shown in Lemma 2.11 of [25], we have that

$$
L^{2}(\Omega, \mathrm{d} \Omega)=\bigoplus_{k=0}^{\infty} \mathcal{H}_{k}
$$

We also let

$$
P_{k}: L^{2}(\Omega, \mathrm{d} \Omega) \rightarrow \mathcal{H}_{k}
$$

be the projection operator onto the $(k+1)$ th eigenspace of $\mathcal{L}_{\sigma}$ defined in (2.4).

The function space $M^{2,2}(\Omega)$ : In order to introduce the notion of solution for Eq. $(0.1)$, it is natural to consider the following function space associated with the Hörmander vector fields

$$
\begin{aligned}
& X_{i}=\partial_{z_{i}}, \quad i=1, \ldots, N . \\
& Y_{j}=z_{j} \partial_{t}, \quad j=1, \ldots, N .
\end{aligned}
$$

We define

$$
\begin{aligned}
M^{2,2}(\Omega)= & \left\{u \in L^{2}(\Omega): X_{i} u, Y_{j} u, X_{i} X_{j} u, X_{i} Y_{j} u, Y_{i} X_{j} u, Y_{i} Y_{j} u\right. \\
& \left.\in L^{2}(\Omega), i, j \in\{1, \ldots, N\}\right\} .
\end{aligned}
$$

Note that, by Theorem 1 in [33],$M^{2,2}(\Omega)$ forms a Hilbert space with respect to the norm

$$
\begin{aligned}
\|u\|_{M^{2,2}(\Omega)}: & =\|u\|_{L^{2}(\Omega)}+\left\|\left|\nabla_{z} u\right|^{2}\right\|_{L^{2}(\Omega)}+\left\||z| \mid \partial_{t} u\right\| \|_{L^{2}(\Omega)} \\
& +\sum_{1 \leq i, j \leq N}\left\|X_{i} X_{j} u\right\|_{L^{2}(\Omega)}+\left\|X_{i} Y_{j} u\right\|_{L^{2}(\Omega)}+\left\|Y_{i} X_{j} u\right\|_{L^{2}(\Omega)} \\
& +\left\|Y_{i} Y_{j} u\right\|_{L^{2}(\Omega)} .
\end{aligned}
$$

Also, from the Sobolev embedding theorem as in [33], it follows that

$$
u, \nabla_{H} u \in L_{l o c}^{2^{*}}(\Omega), \quad 2^{*}=\frac{2(N+2)}{N}
$$

where

$$
\nabla_{H} u=\left(X_{1} u, \ldots, X_{N} u, Y_{1} u, \ldots Y_{N} u\right)
$$




\section{Carleman estimates}

In this section, we first recall a $L^{p}-L^{q}$-type Carleman estimate derived in [25] using which we prove one of our strong unique continuation results as in Theorem 4.2. This corresponds to the situation when the potential $V$ has the growth assumption as in (1.7). We then derive a particular $L^{2}-L^{2}$ Carleman inequality where we obtain a certain asymptotic behaviour of the constant involved in the inequality in terms of the parameter $s$, where $s$ corresponds to the exponent of the singular weight in the Carleman estimate (see Theorem 3.3). Using such an estimate, we argue as in [31] and obtain strong unique continuation property for (0.1) when the potential $V$ satisfies the Hardy-type growth assumption as in (1.8). The reader should note that our proof of this new $L^{2}-L^{2}$ Carleman estimate relies crucially on the following $L^{2}-L^{2}$ estimate for the projection operator $P_{k}$ established in [25] which can be stated as follows.

Theorem 3.1 There exists a constant $C>0$ depending only on $N$ and $\alpha$, such that for any $h \in L^{2}(\Omega, d \Omega)$

$$
\int_{\Omega}\left|\sin ^{-\alpha} \phi P_{k}\left(\sin ^{-\alpha}(.) h\right)(\phi, \omega)\right|^{2} d \Omega \leq C \int_{\Omega}|h|^{2} d \Omega .
$$

Here, $0 \leq \alpha<\frac{1}{2}$, if $N$ is even and $0 \leq \alpha<\frac{3}{8}$, if $N$ is odd.

Next we recall a Carleman inequality derived in [25] (see Theorem 5.1 in [25]), which will be instrumental in proving Theorem 4.2. For the rest of the discussion in this paper, we will denote by $\mathcal{L}$ the Baouendi-Grushin operator on $\mathbb{R}^{N+1}$ as in $(0.1)$ defined by

$$
\mathcal{L}:=\Delta_{z}+|z|^{2} \frac{\partial^{2}}{\partial t^{2}}
$$

Theorem 3.2 Let $0<\delta<\frac{1}{4}, s>100$ and $\operatorname{dist}(s, \mathbb{N})=\frac{1}{2}$. Suppose that $p=\frac{2 N}{N-1}$ and $q=\frac{2 N}{N+1}$. Then there exists constant $C>0$ depending only on $\delta$ and $N$, such that for $f \in C_{c}^{\infty}\left(\mathbb{R}^{N+1} \backslash\{0\}\right)$, the following inequality holds

$$
\left\|\rho^{-s}(\sin \phi)^{\delta} f\right\|_{L^{p}\left(\mathbb{R}^{N+1}, \frac{d z d t}{\rho^{N+2}}\right)} \leq C\left\|\rho^{-s+2}(\sin \phi)^{-\delta} \mathcal{L}(f)\right\|_{L^{q}\left(\mathbb{R}^{N+1}, \frac{d z d t}{\rho^{N+2}}\right)}
$$

if $N \geq 2$ is even, and

$$
\left\|\rho^{-s}(\sin \phi)^{\frac{1}{4 p}+\delta} f\right\|_{L^{p}\left(\mathbb{R}^{N+1}, \frac{d z d t}{\rho^{N+2}}\right)} \leq C\left\|\rho^{-s+2}(\sin \phi)^{-\frac{1}{4 p}-\delta} \mathcal{L}(f)\right\|_{L^{q}\left(\mathbb{R}^{N+1}, \frac{d z d t}{\rho^{N+2}}\right)},
$$

if $N \geq 3$ is odd.

Now with certain modifications (which will be pointed out) in the proof of Theorem 5.1 in [25], we show that the following $L^{2}-L^{2}$ Carleman inequality can be derived. As remarked earlier, the main feature of this inequality is the prescribed asymptotic behaviour of the constant and that is crucially used in the proof of our Theorem 4.3 which concerns the growth assumption on $V$ as in (1.8).

Theorem 3.3 Let $0<\delta<\frac{1}{4}, s>100$ and $\operatorname{dist}(s, \mathbb{N})=\frac{1}{2}$. Then there exists a constant $C>0$ depending only on $\delta$ and $N$, such that for $f \in C_{c}^{\infty}\left(\mathbb{R}^{N+1} \backslash\{0\}\right)$, the following inequality holds, 


$$
\left\|\rho^{-s}(\sin \phi)^{\delta} f\right\|_{L^{2}\left(\mathbb{R}^{N+1}, \frac{d z d t}{\rho^{N+2}}\right)} \leq \frac{C \log _{2} s}{s}\left\|\rho^{-s+2}(\sin \phi)^{-\delta} \mathcal{L}(f)\right\|_{L^{2}\left(\mathbb{R}^{N+1}, \frac{d z d t}{\rho^{N+2}}\right)},
$$

if $N \geq 2$ is even, and

$$
\left\|\rho^{-s}(\sin \phi)^{\frac{1}{8}+\delta} f\right\|_{L^{2}\left(\mathbb{R}^{N+1}, \frac{d z d t}{\rho^{N+2}}\right)} \leq \frac{C \log _{2} s}{s}\left\|\rho^{-s+2}(\sin \phi)^{-\frac{1}{8}-\delta} \mathcal{L}(f)\right\|_{L^{2}\left(\mathbb{R}^{N+1}, \frac{d z d t}{\rho^{N+2}}\right)},
$$

if $N \geq 3$ is odd.

Remark 3.4 We note that for the $L^{2}-L^{2}$ Carleman estimate corresponding to the standard Laplacian, i.e. $\mathcal{L}=\Delta$, it can be shown that the asymptotic behaviour of the constant is in fact $\frac{C}{s}$ as $s \rightarrow \infty$ for some universal $C$ (see, for instance, [1]). This is clearly better than the one we have in Theorem 3.3. However, for our application to unique continuation as in Theorem 4.3 , it turns out that the asymptotic behaviour of the constant that we obtain in Theorem 3.3 suffices.

Proof We will point out the changes only for the case when $N$ is even. In the case of $N$ odd, the proof will follow similarly. In view of the discussion in the proof of Theorem 5.1 in [25] (more precisely, as on pp. 157-158 in [25]), it suffices to show that the following inequality holds

$$
\begin{aligned}
& \left\|R_{S}(g)\right\|_{L^{2}\left(\mathbb{R} \times \Omega,(\sin \phi)^{-1+2 \delta} \mathrm{d} y \mathrm{~d} \Omega\right)} \leq \frac{C \log _{2} s}{s}\|g\|_{L^{2}\left(\mathbb{R} \times \Omega,(\sin \phi)^{-1-2 \delta} \mathrm{d} y \mathrm{~d} \Omega\right)}, \\
& \quad \forall g \in C_{c}^{\infty}(\mathbb{R} \times \Omega) .
\end{aligned}
$$

Here,

$$
R_{S}(g)(y, \phi, \omega)=\int_{\mathbb{R}} \int_{\mathbb{R}} \mathrm{e}^{i(y-x) \eta} \sum_{k=0}^{\infty} \frac{Q_{k}(g)(x, \phi, \omega)}{a_{s}(\eta, k)} \mathrm{d} \eta \mathrm{d} x,
$$

where the operator $Q_{k}$ is defined by

$$
Q_{k}(g)(x, \phi, \omega)=P_{k}\left(\frac{g(x, \cdot, \cdot)}{\sin (\cdot)}\right)(\phi, \omega)
$$

and

$$
\begin{aligned}
a_{s}(\eta, k)= & -\left(\eta-i\left(\left(s+\frac{N+1}{2}\right)-\sqrt{k(N+k)+s+\frac{(N+1)^{2}}{4}}\right)\right) \\
& \cdot\left(\eta-i\left(\left(s+\frac{N+1}{2}\right)+\sqrt{k(N+k)+s+\frac{(N+1)^{2}}{4}}\right)\right) .
\end{aligned}
$$

Now, fix $s>100$ such that $\operatorname{dist}(s, \mathbb{N})=\frac{1}{2}$. Let $m$ satisfies $2^{m} \leq \frac{s}{10}<2^{m+1}$. Similar to [25], we choose a partition of unity $\left\{\Phi_{\beta}\right\}_{\beta=0}^{m}$ for $\mathbb{R}_{+}$such that

$$
\left\{\begin{array}{l}
\sum_{\beta} \Phi_{\beta}(r)=1, \text { for all } r>0 \\
\operatorname{supp} \Phi_{\beta} \subset\left\{r: 2^{\beta-2} \leq r \leq 2^{\beta}\right\}, \beta=1,2, \ldots, m-1 \\
\operatorname{supp} \Phi_{0} \subset\{r: 0<r \leq 1\} \\
\operatorname{supp} \Phi_{m} \subset\left\{r: r \geq \frac{s}{40}\right\}
\end{array}\right.
$$


and

$$
\left|\frac{d^{l}}{\mathrm{~d} r^{l}} \Phi_{\beta}(r)\right| \leq \frac{C_{l}}{2^{\beta l}}, \quad l=0,1,2, \ldots
$$

For $0 \leq \beta \leq m$, we define

$$
b_{s}^{\beta}(\eta, k)=\frac{1}{a_{s}(\eta, k)} \Phi_{\beta}\left(\left|\eta-i\left(\left(s+\frac{N+1}{2}\right)-\sqrt{k(N+k)+s+\frac{(N+1)^{2}}{4}}\right)\right|\right)
$$

and

$$
R_{s}^{\beta}(g)(y, \phi, \omega)=\int_{\mathbb{R}} \int_{\mathbb{R}} \mathrm{e}^{i(y-x) \eta} \sum_{k=0}^{\infty} Q_{k}(g)(x, \phi, \omega) b_{s}^{\beta}(\eta, k) \mathrm{d} \eta \mathrm{d} x .
$$

We further define

$$
F_{s}^{\beta}(x, y, \phi, \omega)=\int_{\mathbb{R}} \mathrm{e}^{i(y-x) \eta} \sum_{k=0}^{\infty} Q_{k}(g)(x, \phi, \omega) b_{s}^{\beta}(\eta, k) \mathrm{d} \eta .
$$

Now, suppose there exists $f_{s}^{\beta} \in L^{1}(\mathbb{R})$ such that

$$
\left\|F_{s}^{\beta}(x, y, \cdot, \cdot)\right\|_{L^{2}\left(\Omega,(\sin \phi)^{-1+2 \delta} \mathrm{d} \Omega\right)} \leq f_{s}^{\beta}(|x-y|)\|g(x, \cdot, \cdot)\|_{L^{2}\left(\Omega,(\sin \phi)^{-1-2 \delta} \mathrm{d} \Omega\right)},
$$

then we can use Minkowski's integral inequality to estimate $\left\|R_{S}^{\beta}(g)\right\|_{L^{2}\left(\mathbb{R} \times \Omega,(\sin \phi)^{-1+2 \delta} \mathrm{d} y \mathrm{~d} \Omega\right)}$ in the following manner

$$
\begin{aligned}
& \left\|R_{s}^{\beta}(g)\right\|_{L^{2}\left(\mathbb{R} \times \Omega,(\sin \phi)^{-1+2 \delta} \mathrm{d} y \mathrm{~d} \Omega\right)} \\
& \left.=\left(\int_{\mathbb{R}}\left(\int_{\Omega}\left(\int_{\mathbb{R}} F_{s}^{\beta}(x, y, \phi, \omega)(\sin \phi)^{\frac{-1+2 \delta}{2}} \mathrm{~d} x\right)^{2} \mathrm{~d} \Omega\right)^{\frac{1}{2}}\right)^{2} \mathrm{~d} y\right)^{\frac{1}{2}} \\
& \quad \leq\left(\int_{\mathbb{R}}\left(\int_{\mathbb{R}}\left\|F_{S}^{\beta}(x, y, \cdot, \cdot)\right\|_{L^{2}\left(\Omega,(\sin \phi)^{-1+2 \delta} \mathrm{d} \Omega\right)} \mathrm{d} x\right)^{2} \mathrm{~d} y\right)^{\frac{1}{2}} \\
& \quad \leq\left(\int_{\mathbb{R}}\left(\int_{\mathbb{R}} f_{s}^{\beta}(|x-y|)\|g(x, \cdot, \cdot)\|_{L^{2}\left(\Omega,(\sin \phi)^{-1-2 \delta} \mathrm{d} \Omega\right)} \mathrm{d} x\right)^{2} \mathrm{~d} y\right)^{\frac{1}{2}} \\
& \quad \leq\left\|f_{s}^{\beta}\right\|_{L^{1}(\mathbb{R})}\|g\|_{L^{2}\left(\mathbb{R} \times \Omega,(\sin \phi)^{-1-2 \delta} \mathrm{d} y \mathrm{~d} \Omega\right)}
\end{aligned}
$$

where to get the last two inequalities we have used (3.16) and the Young's inequality for convolution, respectively. Now since

$$
R_{S}=\sum_{\beta=0}^{m} R_{s}^{\beta},
$$

therefore the preceding inequality clearly shows that to establish (3.7) we only need to find appropriate $f_{s}^{\beta}$ in (3.16). 
First consider $0 \leq \beta \leq m-1$. In this case, if $b_{s}^{\beta}(\eta, k) \neq 0$, then by (3.11),

$$
\delta 2^{\beta-2} \leq\left|\eta-i\left(\left(s+\frac{N+1}{2}\right)-\sqrt{k(N+k)+s+\frac{(N+1)^{2}}{4}}\right)\right| \leq 2^{\beta} .
$$

Hence, $|\eta| \leq 2^{\beta}$ and $|s-k| \leq 2^{\beta+1}$. Therefore, there are at most $2^{\beta+2}$ nonzero terms in the sum over $k$ in (3.13) and one can compare the values of these $k^{\prime}$ s to $s$. So as in (5.19) in [25], using (3.12), (3.10) and (3.13) we conclude that

$$
\left|\frac{\partial^{j}}{\partial \eta^{j}} b_{s}^{\beta}(\eta, k)\right| \leq \frac{C_{j} 2^{-\beta}}{s 2^{j \beta}} .
$$

Invoking (3.1) with $h=(\sin \phi)^{\alpha-1} g$, we get

$$
\left\|Q_{k}(g)\right\|_{L^{2}\left(\Omega,(\sin \phi)^{-2 \alpha} \mathrm{d} \Omega\right)} \leq C\|g\|_{L^{2}\left(\Omega,(\sin \phi)^{2 \alpha-2} \mathrm{~d} \Omega\right)},
$$

for $0<\alpha<\frac{1}{2}$. Choosing $\delta=\frac{1}{2}-\alpha$ in the last inequality, we get

$$
\left\|Q_{k}(g)\right\|_{L^{2}\left(\Omega,(\sin \phi)^{-1+2 \delta} \mathrm{d} \Omega\right)} \leq C\|g\|_{L^{2}\left(\Omega,(\sin \phi)^{-1-2 \delta} \mathrm{d} \Omega\right)}
$$

Now performing integration by parts and using (3.18), (3.19), we conclude that

$$
\begin{aligned}
& \left\|F_{s}^{\beta}(x, y, \cdot, \cdot)\right\|_{L^{2}\left(\Omega,(\sin \phi)^{-1+2 \delta} \mathrm{d} \Omega\right)} \\
& \quad \leq \frac{C}{|y-x|^{j}} \sum_{k=0}^{\infty} \int_{\mathbb{R}}\left|\left(\frac{\partial}{\partial \eta}\right)^{j} b_{s}^{\beta}(\eta, k)\right| \mathrm{d} \eta\left\|Q_{k}(g)\right\|_{L^{2}\left(\Omega,(\sin \phi)^{-1+2 \delta} \mathrm{d} \Omega\right)} \\
& \leq \frac{C 2^{\beta}}{s\left(2^{\beta}|y-x|\right)^{j}}\|g\|_{L^{2}\left(\Omega,(\sin \phi)^{-1-2 \delta} \mathrm{d} \Omega\right)} .
\end{aligned}
$$

In the above estimate, we crucially used the fact that the support of integral lies in $\{|\eta| \leq \beta\}$ and the fact that at most $2^{\beta+2}$ terms survive in the above summation over $k$.

If we now choose $j=10$ and $j=0$, then we obtain

$$
\left\|F_{s}^{\beta}(x, y, \cdot, \cdot)\right\|_{L^{2}\left(\Omega,(\sin \phi)^{-1+2 \delta} \mathrm{d} \Omega\right)} \leq \frac{C}{s\left(1+2^{\beta}|y-x|\right)^{10}} 2^{\beta}\|g\|_{L^{2}\left(\Omega,(\sin \phi)^{-1-2 \delta} \mathrm{d} \Omega\right)} .
$$

Thus, the inequality above can be casted in the form as in (3.16), provided we take $f_{s}^{\beta}(r)=$ $\frac{C}{s\left(1+2^{\beta}|r|\right)^{10}} 2^{\beta}$. Clearly with such a choice of $f_{s}^{\beta}$, we have that the following estimate holds,

$$
\left\|f_{s}^{\beta}\right\|_{L^{1}(\mathbb{R})} \leq \frac{C}{s}
$$

Then from the estimates as in (3.17), we have that for $\beta=0, \ldots m-1$, the following inequality holds,

$$
\left\|R_{S}^{\beta}(g)\right\|_{L^{2}\left(\mathbb{R} \times \Omega,(\sin \phi)^{-1+2 \delta} \mathrm{d} y \mathrm{~d} \Omega\right)} \leq \frac{C}{S}\|g\|_{L^{2}\left(\mathbb{R} \times \Omega,(\sin \phi)^{-1-2 \delta} \mathrm{d} y \mathrm{~d} \Omega\right)} .
$$

Therefore by summing over $\beta=0$ to $\beta=m-1$ and by using the fact that

$$
m \leq \log _{2} s
$$


we obtain

$$
\sum_{\beta=0}^{m-1}\left\|R_{s}^{\beta}(g)\right\|_{L^{2}\left(\mathbb{R} \times \Omega,(\sin \phi)^{-1+2 \delta} \mathrm{d} y \mathrm{~d} \Omega\right)} \leq \frac{C \log _{2} S}{s}\|g\|_{L^{2}\left(\mathbb{R} \times \Omega,(\sin \phi)^{-1-2 \delta} \mathrm{d} y \mathrm{~d} \Omega\right)} .
$$

Finally, we consider the case of $\beta=m$. For this, we observe that on the support of $b_{s}^{m}(\eta, k)$ we have

$$
\left|a_{s}(\eta, k)\right| \sim(|\eta|+s+k)^{2}
$$

and also

$$
\left|\left(\frac{\partial}{\partial \eta}\right)^{j} b_{s}^{N}(\eta, k)\right| \leq \frac{C_{j}}{\left(|\eta|^{2}+k+s\right)^{j+2}} .
$$

Similarly as in the previous case, by integration by parts, using and (3.22) and (3.23) and by choosing $j=0$ and $j=2$ we arrive at

$$
\begin{aligned}
& \left\|F_{s}^{m}(x, y, \cdot, \cdot)\right\|_{L^{2}\left(\Omega,(\sin \phi)^{-1-2 \epsilon} \mathrm{d} \Omega\right)} \\
& \quad \leq \sum_{k=0}^{\infty} \frac{C}{(k+s)\left(1+(k+s)^{2}|y-x|^{2}\right)}\|g\|_{L^{2}\left(\Omega,(\sin \phi)^{-1-2 \epsilon} \mathrm{d} \Omega\right)} .
\end{aligned}
$$

Thus we are again in the situation as in (3.16) with $f_{s}^{\beta}(r)=\sum_{k=0}^{\infty} \frac{C}{(k+s)\left(1+(k+s)^{2}|r|^{2}\right)}$. Clearly,

$$
\left\|f_{s}^{\beta}\right\|_{L^{1}(\mathbb{R})} \leq C \sum_{k=0}^{\infty} \frac{1}{(k+s)^{2}} \leq \frac{C}{s} .
$$

Therefore, we conclude that

$$
\left\|R_{s}^{m}(g)\right\|_{L^{2}\left(\mathbb{R} \times \Omega,(\sin \phi)^{-1+2 \delta} \mathrm{d} y \mathrm{~d} \Omega\right)} \leq \frac{C}{S}\|g\|_{L^{2}\left(\mathbb{R} \times \Omega,(\sin \phi)^{-1-2 \delta} \mathrm{d} y \mathrm{~d} \Omega\right)}
$$

Finally, combining (3.21) and (3.25) we obtain (3.7). This completes the proof.

\section{Strong unique continuation}

In this section, we establish the strong unique continuation property for $(0.1)$ with the growth assumptions on $V$ as in (1.2) or (1.4) using the Carleman estimates as stated in Theorems 3.2 and 3.3. For $r>0$ and $t_{0} \in \mathbb{R}$, we define

$$
B_{r}\left(\left(0, t_{0}\right)\right):=\left\{(z, t) \in \mathbb{R}^{N+1}:\left(|z|^{4}+4\left|t-t_{0}\right|^{2}\right)^{\frac{1}{4}}<r\right\}, \quad B_{r}:=B_{r}((0,0)) .
$$

Definition 4.1 We recall that $u$ vanishes to infinite order at the point $\left(0, t_{0}\right)$ in the $L^{p}$ mean, if

$$
\int_{B_{r}\left(0, t_{0}\right)}|u|^{p} \mathrm{~d} z \mathrm{~d} t=\mathcal{O}\left(r^{l}\right), \quad \text { as } r \rightarrow 0 \text { for all } l>0 .
$$


We note that in [18] as well as in [21], the authors insisted in their definition of vanishing to infinite order at $(0,0)$ that the function $u$ must satisfy the following weaker assumption

$$
\int_{B_{r}} u^{2} \psi \mathrm{d} z \mathrm{~d} t=O\left(r^{l}\right), \quad \text { for all } l>0 \text { as } r \rightarrow 0^{+}
$$

It, however, turns out that for functions in $M^{2,2}\left(B_{1}\right)$, vanishing to infinite order as in (4.2) (for $p=2$ ) is in fact equivalent to vanishing to infinite order in the sense of (4.3). This is the content of the next lemma.

Lemma 4.1 Let $u \in M^{2,2}\left(B_{1}\right)$. Then $u$ vanishes to infinite order in the $L^{2}$ mean at $(0,0)$ (in the sense of Definition 4.1) if and only if $u$ vanishes to infinite order in the sense of (4.3).

Proof First we note that if $u$ vanishes to infinite order in the $L^{2}$ mean, since $\psi \leq 1$, we have

$$
\int_{B_{r}} u^{2} \psi \mathrm{d} z \mathrm{~d} t \leq \int_{B_{r}} u^{2} \mathrm{~d} z \mathrm{~d} t
$$

and hence it follows that $u$ vanishes to infinite order in the sense of (4.3). Now let us look at the converse implication. First we note that from (2.14) we have

$$
u \in L_{l o c}^{2^{*}}\left(B_{1}\right), \quad \text { where } 2^{*}=\frac{2(N+2)}{N}
$$

Now if $u$ vanishes to infinite order in the sense of (4.3), it follows from the interpolation inequality

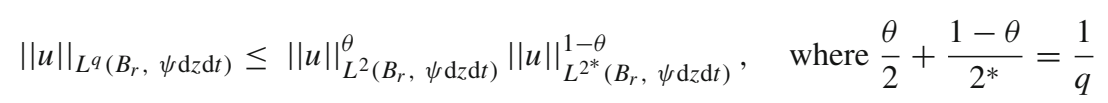

that for all $q<2^{*}$, we have

$$
\int_{B_{r}} u^{q} \psi \mathrm{d} z \mathrm{~d} t=O\left(r^{l}\right), \quad \text { as } r \rightarrow 0^{+}
$$

for all $l>0$. Now we fix some $q \in\left(2,2^{*}\right)$. Then, from the Hölder inequality it follows

$$
\int_{B_{r}}|u| \mathrm{d} z \mathrm{~d} t \leq\left(\int_{B_{r}}|u|^{q} \psi \mathrm{d} z \mathrm{~d} t\right)^{1 / q}\left(\int_{B_{r}} \psi^{-\frac{1}{q-1}} \mathrm{~d} z \mathrm{~d} t\right)^{\frac{q-1}{q}}
$$

Now since $q>2$, therefore we have that $\frac{1}{q-1}<1$ and hence it follows from the polar decomposition as in (2.3) that

$$
\int_{B_{r}} \psi^{-\frac{1}{q-1}} \mathrm{~d} z \mathrm{~d} t<\infty, \text { Note that } \psi=\sin \phi
$$

Therefore, combining (4.4) and (4.5) we get that

$$
\int_{B_{r}}|u| \mathrm{d} z \mathrm{~d} t=O\left(r^{k}\right), \quad \text { as } r \rightarrow 0
$$

for all $k>0$. Now again by using an interpolation inequality of the type

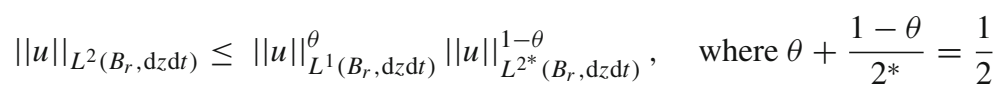

it follows from (4.6) that $u$ vanishes to infinite order in the $L^{2}$ mean as in Definition 4.1. The claim in the lemma thus follows. 
Our first unique continuation result can now be stated as follows.

Theorem 4.2 With $\mathcal{L}$ as in (3.2), let $u \in M^{2,2}\left(B_{r_{0}}\right)$ for some $r_{0}>0$ be a solution to

$$
\mathcal{L} u=V u \quad \text { in } B_{r_{0}},
$$

for a potential $V$ satisfying (1.7). If $u$ vanishes to infinite order at $(0,0)$ in the sense of (4.3), then $u \equiv 0$ in $B_{r_{0}}$.

Proof The proof of this result uses the Carleman estimates as in Theorem 3.2. First we note that in view of Lemma 4.1, we have that $u$ vanishes to infinite order in the $L^{2}$ mean at $(0,0)$. Now as in [25], we let $\xi \in C_{c}^{\infty}\left(\mathbb{R}^{N+1}\right)$ such that $\xi=1$ when $\rho(z, t) \leq \frac{1}{2}$ and $\xi=0$ when $\rho(z, t) \geq \frac{3}{4}$. Also, we define $\Psi_{j}(\rho)=\Psi(j \rho)$, where $\Psi=1-\xi$.

We first consider the case when $N$ is even. Without loss of generality, we may assume for simplicity that $r_{0}=1$. Using a standard limiting argument (i.e. by approximation with smooth functions), one can show that the Carleman estimate (3.3) holds for $f=\xi \Psi_{j} u$. This gives

$$
\begin{aligned}
\left\|\rho^{-s}(\sin \phi)^{\delta} \xi \Psi_{j} u\right\| \|_{L^{p}\left(\mathbb{R}^{N+1}, \frac{\mathrm{d} z \mathrm{~d} t}{\rho^{N+2}}\right)} \leq & C\left\|\rho^{-s+2}(\sin \phi)^{-\delta} \mathcal{L}\left(\Psi_{j} u\right)\right\|_{L^{q}\left(B_{r}, \frac{\mathrm{d} \mathrm{z} t}{\rho^{N+2}}\right)} \\
& +\left\|\rho^{-s+2}(\sin \phi)^{-\delta} \mathcal{L}(\xi u)\right\|_{L^{q}\left(\rho \geq r, \frac{\mathrm{d} z \mathrm{~d} t}{\rho^{N+2}}\right)} \\
:= & I+I I
\end{aligned}
$$

where $0<\frac{3}{4 j}<r<\frac{1}{2}$ are constants to be chosen later. For $\delta$ small enough, by using Hölder inequality and (2.3) we conclude that

$$
I I \leq C r^{-s+2-\frac{N+2}{q}}\|\mathcal{L}(\xi u)\|_{L^{2}\left(B_{1}\right)} \leq C r^{-s+2-\frac{N+2}{q}}\|u\|_{M^{2,2}\left(B_{1}\right)} .
$$

Next, we estimate $I$. For this we note that,

$$
\mathcal{L}\left(\Psi_{j} u\right)=\mathcal{L}\left(\Psi_{j}\right) u+2 \nabla_{z} \Psi_{j} \cdot \nabla_{z} u+2|z|^{2} \partial_{t} \Psi_{j} \cdot \partial_{t} u+\Psi_{j} \mathcal{L}(u) .
$$

Now we note that the derivatives of $\Psi_{j}$ are supported in $B_{\frac{3}{4 j}}-B_{\frac{1}{2 j}}$ and satisfy the following bounds

$$
\left|\nabla \Psi_{j}\right|,\left|\nabla^{2} \Psi_{j}\right| \leq C_{0} j^{k} \quad \text { for some } C_{0} \text { universal and some } k
$$

Therefore, by using (4.11), (4.12), Eq. (4.7) satisfied by $u$ and an application of Hölder inequality for $\delta$ small enough, we can estimate I as follows

$$
\begin{aligned}
I \leq & C\left\|\rho^{-s+2}(\sin \phi)^{-\delta} V \Psi_{j} u\right\|_{L^{q}\left(B_{r}, \frac{\mathrm{d} \mathrm{d} t}{\rho^{N+2}}\right)}+C j^{M}\left(\int_{B_{\frac{3}{4 j}}-B_{\frac{1}{2 j}}}|u|^{2} \mathrm{~d} z \mathrm{~d} t\right)^{\frac{1}{2}} \\
& +C j^{M}\left(\int_{B_{\frac{3}{4 j}}-B_{\frac{1}{2 j}}}\left(\left|\nabla_{z} u\right|^{2}+|z|^{2}\left|\partial_{t} u\right|^{2}\right) \mathrm{d} z \mathrm{~d} t\right)^{\frac{1}{2}}
\end{aligned}
$$

where $M>0$ is a constant which depends on $s$. Now using the following variant of the Caccioppoli inequality

$$
\int_{B_{d}-B_{d / 2}}\left(\left|\nabla_{z} u\right|^{2}+|z|^{2}\left|\partial_{t} u\right|^{2}\right) \mathrm{d} z \mathrm{~d} t \leq \frac{C}{d^{2}} \int_{B_{2 d}-B_{d / 4}}\left(u^{2}+|V||u|^{2}\right) \mathrm{d} z \mathrm{~d} t, \quad d>0,
$$


the vanishing to infinite-order property of $u$ and the growth assumption on $V$ as in (1.7), we can conclude that

$$
\begin{aligned}
& C j^{M}\left(\int_{B_{\frac{3}{4 j}}-B_{\frac{1}{2 j}}}|u|^{2} \mathrm{~d} z \mathrm{~d} t\right)^{\frac{1}{2}} \\
& \quad+C j^{M}\left(\int_{B_{\frac{3}{4 j}}-B_{\frac{1}{2 j}}}\left(\left|\nabla_{z} u\right|^{2}+|z|^{2}\left|\partial_{t} u\right|^{2}\right) \mathrm{d} z \mathrm{~d} t\right)^{\frac{1}{2}} \rightarrow 0 \text { as } j \rightarrow \infty
\end{aligned}
$$

Also, using Hölder inequality ( since $\frac{1}{p}=\frac{1}{q}-\frac{1}{N}$ ), we can estimate the quantity

$$
\left\|\rho^{-s+2}(\sin \phi)^{-\delta} V \Psi_{j} u\right\|_{L^{q}\left(B_{r}, \frac{\mathrm{d} z \mathrm{~d} t}{\rho^{N+2}}\right)}
$$

in (4.13) using the growth assumption on $V$ as in (1.7) in the following way,

$$
\begin{aligned}
& C\left\|\rho^{-s+2}(\sin \phi)^{-\delta} V \Psi_{j} u\right\|_{L^{q}\left(B_{r}, \frac{\mathrm{d} z \mathrm{~d} t}{\rho^{N+2}}\right)} \\
& \quad \leq C\left\|(\sin \phi)^{-2 \delta} f(\rho)\right\|_{L^{N}\left(B_{r}, \frac{\mathrm{d} z \mathrm{~d} t}{\rho^{N+2}}\right)}\left\|\rho^{-s}(\sin \phi)^{\delta} \Psi_{j} u\right\|_{L^{p}\left(B_{r}, \frac{\mathrm{d} z \mathrm{~d} t}{\rho^{N+2}}\right)}
\end{aligned}
$$

Therefore, plugging (4.16) and (4.15) into (4.13) and then also by using the estimate for II as in (4.10), we get from (4.8) (after passing $j \rightarrow \infty$ ) that the following inequality holds

$$
\begin{aligned}
& \left\|\rho^{-s}(\sin \phi)^{\delta} u\right\|_{L^{p}\left(B_{r}, \frac{\mathrm{d} z \mathrm{~d} t}{\rho^{N+2}}\right)} \\
& \quad \leq C\left\|(\sin \phi)^{-2 \delta} f(\rho)\right\|_{L^{N}\left(B_{r}, \frac{\mathrm{d} z \mathrm{~d} t}{\rho^{N+2}}\right)}\left\|\rho^{-s}(\sin \phi)^{\delta} u\right\|_{L^{p}\left(B_{r}, \frac{\mathrm{d} z \mathrm{~d} t}{\rho^{N+2}}\right)} \\
& \quad+C r^{-s+2-\frac{N+2}{q}}\|u\|_{M^{2,2}\left(B_{1}\right)} .
\end{aligned}
$$

Now using $\mathrm{d} z \mathrm{~d} t=\frac{1}{2}(\sin \phi)^{\frac{N-2}{2}} \rho^{N+1} \mathrm{~d} \rho \mathrm{d} \phi \mathrm{d} \omega$, we get

$$
\begin{aligned}
& \left\|(\sin \phi)^{-2 \delta} f(\rho)\right\|_{L^{N}\left(B_{r}, \frac{\mathrm{d} z \mathrm{~d} t}{\rho^{N+2}}\right)}=\left(\frac{1}{2} \int_{0}^{r} \frac{f(\rho)^{N}}{\rho} \mathrm{d} \rho \int_{S^{N-1}} \mathrm{~d} \omega \int_{0}^{\pi}(\sin \phi)^{\left.-2 \delta N+\frac{N-2}{2} \mathrm{~d} \phi\right)^{1 / N}}\right. \\
& \leq\left(\frac{1}{2} \int_{0}^{r} \frac{f(\rho)}{\rho} \mathrm{d} \rho \int_{S^{N-1}} \mathrm{~d} \omega \int_{0}^{\pi}(\sin \phi)^{-2 \delta N+\frac{N-2}{2}} \mathrm{~d} \phi\right)^{1 / N},
\end{aligned}
$$

for $r$ small enough such that $f(r) \leq 1$.

At this point using Dini integrability of $f$ as in (1.3), it follows from (4.18) that if we choose $r, \delta$ small enough, then one can ensure that

$$
C\left\|(\sin \phi)^{-2 \delta} f(\rho)\right\|_{L^{N}\left(B_{r}, \frac{\mathrm{d} z \mathrm{~d} t}{\rho^{N+2}}\right)}<\frac{1}{2}
$$

and therefore the term $C\left\|(\sin \phi)^{-2 \delta} f(\rho)\right\|_{L^{N}\left(B_{r}, \frac{\mathrm{d} z \mathrm{~d} t}{\rho^{N+2}}\right)}\left\|\rho^{-s}(\sin \phi)^{\delta} u\right\|_{L^{p}\left(B_{r}, \frac{\mathrm{d} z \mathrm{~d} t}{\rho^{N+2}}\right)}$ in (4.17) can be absorbed in the left-hand side. Consequently, we obtain

$$
\left\|\left(\frac{\rho}{r}\right)^{-s}(\sin \phi)^{\delta} u\right\|_{L^{p}\left(B_{r}, \frac{\mathrm{d} z \mathrm{~d} t}{\rho^{N+2}}\right)} \leq C\|u\|_{M^{2,2}\left(B_{1}\right)} .
$$


Now with $s=k+\frac{1}{2}$ by letting $k \rightarrow \infty$, we conclude $u \equiv 0$ in $B_{r}$. Outside of $B_{r}$, we have that $V$ is bounded and therefore we can now apply the results in [25] to conclude that $u \equiv 0$ in $B_{1}$.

To handle the case when $N \geq 3$ is odd, we instead use the Carleman estimates as in (3.4) similar to that in the proof of Theorem 6.4 in [25]. Then by repeating the same arguments as above, and the fact that

$$
\left\|(\sin \phi)^{-\frac{1}{4 p}-\delta} v\right\|_{L^{q}\left(B_{r}\right)} \leq C\|v\|_{L^{2}\left(B_{r}\right)} \text { for } \delta \text { small }
$$

which follows from Hölder inequality, we obtain by a limiting argument as before

$$
\begin{aligned}
& \left\|\rho^{-s}(\sin \phi)^{\frac{1}{4 p}+\delta} u\right\|_{L^{p}\left(B_{r}, \frac{\mathrm{d} z \mathrm{~d} t}{\rho^{N+2}}\right)} \\
& \leq C\left\|(\sin \phi)^{-\frac{1}{2 p}-2 \delta} f(\rho)\right\|\left\|_{L^{N}\left(B_{r}, \frac{\mathrm{d} z \mathrm{~d} t}{\rho^{N+2}}\right)}\right\| \rho^{-s}(\sin \phi)^{\frac{1}{4 p}+\delta} u \|_{L^{p}\left(B_{r}, \frac{\mathrm{d} z \mathrm{~d} t}{\rho^{N+2}}\right)} \\
& \quad+C r^{-s+2-\frac{N+2}{q}}\|u\|_{M^{2,2}\left(B_{1}\right)}
\end{aligned}
$$

Now again by using polar coordinates, we observe that for $\delta$ small enough

$$
\left\|(\sin \phi)^{-\frac{1}{2 p}-2 \delta} f(\rho)\right\|_{L^{N}\left(B_{r}, \frac{\mathrm{d} z \mathrm{~d} t}{\rho^{N+2}}\right)}=o(1), \quad \text { as } r \rightarrow 0 .
$$

Therefore from (4.20) and (4.21), a careful imitation of the previous proof will yield

$$
\left\|\rho^{-s}(\sin \phi)^{\frac{1}{4 p}+\delta} u\right\|_{L^{p}\left(B_{r}, \frac{\mathrm{d} z \mathrm{~d} t}{\rho^{N+2}}\right)} \leq C r^{-s+2-\frac{N+2}{q}}\|u\|_{M^{2,2}\left(B_{1}\right)} .
$$

From here, we can use the same arguments as in the case of $N \geq 2$ even to conclude the result for the case of $N \geq 3$ odd. This completes the proof.

The next unique continuation result concerns the Hardy-type growth assumption for the potential $V$ as in (1.8).

Theorem 4.3 With $\mathcal{L}$ as in (3.2), let $N$ be even, $r_{0}>0$ and $u \in M^{2,2}\left(B_{r_{0}}\right)$ be a solution to

$$
\mathcal{L} u=V u \text { in } B_{r_{0}},
$$

for a potential $V$ satisfying (1.8) for some $\epsilon>0$. If $u$ vanishes to infinite order at $(0,0)$ in the sense of (4.3), then $u \equiv 0$ in $B_{r_{0}}$.

Proof As in the proof of Theorem 4.2, we may assume $r_{0}=1$ and also that $u$ vanishes to infinite order at $(0,0)$ in the $L^{2}$ mean. Note that the later fact follows from Lemma 4.1. We now proceed as in the proof of Theorem 4.2 and consider the same cut-off functions $\Psi_{j}$ and $\xi$. Now by applying the Carleman estimate as in (3.5) with the $f=\xi \Psi_{j} u$ and $\delta=\frac{\epsilon}{2}$ we obtain

$$
\begin{aligned}
& \left\|\rho^{-s}(\sin \phi)^{\frac{\epsilon}{2}} \xi \Psi_{j} u\right\|_{L^{2}\left(\mathbb{R}^{N+1}, \frac{\mathrm{d} z \mathrm{~d} t}{\rho^{N+2}}\right)} \\
& \quad \leq \frac{C \log _{2} s}{s}\left\|\rho^{-s+2}(\sin \phi)^{-\frac{\epsilon}{2}} \mathcal{L}\left(\Psi_{j} u\right)\right\|_{L^{2}\left(B_{r}, \frac{\mathrm{d} z \mathrm{~d} t}{\rho^{N+2}}\right)} \\
& \quad+\frac{C \log _{2} s}{s}\left\|\rho^{-s+2}(\sin \phi)^{-\frac{\epsilon}{2}} \mathcal{L}(\xi u)\right\|_{L^{2}\left(\rho \geq r, \frac{\mathrm{d} z \mathrm{~d} t}{\rho^{N+2}}\right)}:=I+I I,
\end{aligned}
$$


where $j$ is large enough and $r$ is small enough satisfying $0<\frac{3}{4 j}<r<\frac{1}{2}$. Clearly,

$$
I I \leq \frac{C \log _{2} s}{s} r^{-s+2}\left\|(\sin \phi)^{-\frac{\epsilon}{2}} \mathcal{L}(\xi u)\right\|_{L^{2}\left(\rho \geq r, \frac{\mathrm{d} z \mathrm{~d} t}{\rho^{N+2}}\right)} .
$$

Observe that in the preceding inequality, the term involving the $L^{2}$ norm is finite, i.e.

$$
\left\|(\sin \phi)^{-\frac{\epsilon}{2}} \mathcal{L}(\xi u)\right\|_{L^{2}\left(\rho \geq r, \frac{\mathrm{d} z \mathrm{~d} t}{\rho^{N+2}}\right)}<\infty .
$$

This can be seen as follows. We first note that away from the origin, we have that $V$ is bounded. Therefore, since $u$ satisfies (4.22), it follows from the De Giorgi-Nash-Moser theory in this setting (see, for instance, [10]) that $u$ is bounded away from the origin and consequently the same holds for $\mathcal{L}(u)$ as well because of (4.22). Therefore, using the boundedness of $u, \mathcal{L}(u)$, higher integrability of $\nabla_{z} u,|z| \partial_{t} u$ [as in (2.14)], Hölder inequality and the fact that

$$
\int_{\{\rho \geq r\}}(\sin \phi)^{-\delta} \mathrm{d} z \mathrm{~d} t<\infty,
$$

for any $\delta \in(0,1)$, we can assert that the term involving $L^{2}$ norm in (4.24) is finite for small enough $\epsilon$. Note that in the growth assumption (1.8), since $\psi=\sin \phi \leq 1$, therefore in the very first place we can assume that (1.8) holds for $\epsilon$ small enough.

Next we estimate $I$. Again from the following interpolation inequality

$$
\|u\|_{L^{q}} \leq\|u\|_{L^{2}}^{\theta}\|u\|_{L^{2^{*}}}^{1-\theta}, \quad \text { where } \frac{\theta}{2}+\frac{1-\theta}{2^{*}}=\frac{1}{q}
$$

and also by using (2.14) we can infer that since $u$ vanishes to infinite order at $(0,0)$ in the $L^{2}$ mean, it also vanishes of infinite order in the $L^{q}$ mean for any $q \in\left(2,2^{*}\right)$. Similarly by using the variant of the Caccioppoli inequality as in (4.14) and interpolation inequality as above, we can assert $\left|\nabla_{z} u\right|$ and $|z|\left|\partial_{t} u\right|$ vanish to infinite order at $(0,0)$ in the $L^{q}$ mean for any $q \in\left(2,2^{*}\right)$. Therefore, combining these with the fact that

$$
\int_{B_{1}}(\sin \phi)^{-\delta} \mathrm{d} z \mathrm{~d} t<\infty, \quad \forall \delta \in(0,1),
$$

we can conclude that $(\sin \phi)^{-\epsilon}|u|^{2}$ and $(\sin \phi)^{-\epsilon}\left(\left|\nabla_{z} u\right|^{2}+|z|^{2}\left|\partial_{t} u\right|^{2}\right)$ vanish to infinite order in the $L^{1}$ mean at the point $(0,0)$ for small enough $\epsilon$. Hence, for some large enough $M$ depending on $s$ we have,

$$
\begin{aligned}
I \leq & \frac{C \log _{2} s}{s}\left\|\rho^{-s+2}(\sin \phi)^{-\frac{\epsilon}{2}} V \Psi_{j} u\right\|_{L^{2}\left(B_{r}, \frac{\mathrm{d} z \mathrm{~d} t}{\rho^{N+2}}\right)} \\
& +\frac{C \log _{2} s}{s} j^{M}\left(\int_{B_{\frac{3}{4 j}} \frac{1}{2 j}}(\sin \phi)^{-\epsilon}|u|^{2} \mathrm{~d} z \mathrm{~d} t\right)^{\frac{1}{2}} \\
& +\frac{C \log _{2} s}{s} j^{M}\left(\int_{B_{\frac{3}{4 j}}-\frac{1}{2 j}}(\sin \phi)^{-\epsilon}\left(\left|\nabla_{z} u\right|^{2}+|z|^{2}\left|\partial_{t} u\right|^{2}\right) \mathrm{d} z \mathrm{~d} t\right)^{\frac{1}{2}} \\
\leq & \frac{C \log _{2} s}{s}\left\|\rho^{-s}(\sin \phi)^{\frac{\epsilon}{2}} \Psi_{j} u\right\|_{L^{2}\left(B_{r}, \frac{\mathrm{d} \mathrm{d} t t}{\rho^{N+2}}\right)}+o(1), \quad \text { as } j \rightarrow \infty .
\end{aligned}
$$


To achieve the last inequality we have used the imposed condition (1.8) on $V$. Therefore, letting $j \rightarrow \infty$ and making use of inequalities (4.24), (4.26) we conclude from (4.23) that the following holds

$$
\begin{aligned}
\left\|\rho^{-s}(\sin \phi)^{\frac{\epsilon}{2}} u\right\|_{L^{2}\left(B_{r}, \frac{\mathrm{d} z \mathrm{~d} t}{\rho^{N+2}}\right)} \leq & \frac{C \log _{2} s}{s}\left\|\rho^{-s}(\sin \phi)^{\frac{\epsilon}{2}} u\right\|_{L^{2}\left(B_{r}, \frac{\mathrm{d} z \mathrm{~d} t}{\rho^{N+2}}\right)} \\
& +\frac{C \log _{2} s}{s} r^{-s+2}\left\|(\sin \phi)^{-\frac{\epsilon}{2}} \mathcal{L}(\xi u)\right\|_{L^{2}\left(\rho \geq r, \frac{\mathrm{d} z \mathrm{~d} t}{\rho^{N+2}}\right)} .
\end{aligned}
$$

Now letting $s=s_{k}:=k+\frac{1}{2}$, for large enough $k$ we have

$$
\frac{C \log _{2} s_{k}}{s_{k}}<\frac{1}{2}
$$

and therefore in the preceding inequality, for large enough $k$, the first term can be absorbed in the left-hand side, and consequently, we will have

$$
\left\|\left(\frac{\rho}{r}\right)^{-s_{k}}(\sin \phi)^{\frac{\epsilon}{2}} u\right\|_{L^{2}\left(B_{r}, \frac{\mathrm{d} z \mathrm{~d} t}{\rho^{N+2}}\right)} \leq \frac{C \log _{2} s_{k}}{s_{k}} r^{2}\left\|(\sin \phi)^{-\frac{\epsilon}{2}} \mathcal{L}(\xi u)\right\|_{L^{2}\left(\rho \geq r, \frac{\mathrm{d} z \mathrm{~d} t}{\rho^{N+2}}\right)} .
$$

Now in view of (4.25), by passing to limit $k \rightarrow \infty$ in (4.27) we can assert that $u \equiv 0$ in $B_{r}$. The rest of the argument remains the same as in the proof of the previous theorem, and hence, we can again conclude that $u \equiv 0$ in $B_{1}$.

Now in the case when $N \geq 3$ is odd, because the nature of the Carleman estimate in (3.4) is different, therefore we cannot assert that Theorem 4.3 holds. However using the estimate in (3.4) and a slight adaptation of the previous proof, we can assert that the following unique continuation property holds.

Theorem 4.4 With $\mathcal{L}$ as in (3.2), let $N \geq 3$ be odd, $r_{0}>0$ and $u \in M^{2,2}\left(B_{r_{0}}\right)$ be a solution to

$$
\mathcal{L} u=V u \text { in } B_{r_{0}},
$$

for a potential $V$ satisfying

$$
|V(z, t)| \leq \frac{C(\sin \phi)^{\frac{1}{4}+\epsilon}}{\rho(z, t)^{2}}
$$

for some $\epsilon>0$. If $u$ vanishes to infinite order at $(0,0)$ in the sense of $(4.3)$, then $u \equiv 0$ in $B_{r_{0}}$.

\section{Application to unique continuation on the Heisenberg group $\mathbb{H}^{n}$}

We recall that on the Heisenberg group $\mathbb{H}^{n}=\mathbb{R}^{2 n+1}$, if we denote an arbitrary point by $(x, y, t)=\left(x_{1}, \ldots x_{n}, y_{1}, \ldots y_{n}, t\right)$, then the group operation is defined as follows

$$
(x, y, t) \circ\left(x^{\prime}, y^{\prime}, t^{\prime}\right)=\left(x+x^{\prime}, y+y^{\prime}, t+t^{\prime}+2\left(x^{\prime} \cdot y-x \cdot y^{\prime}\right)\right)
$$

Let $z=(x, y)$. Note that the horizontal vector space $V_{1}$ is spanned by

$$
X_{i}=\partial_{x_{i}}+2 y_{i} \partial_{t}, \quad i=1, \ldots, n
$$




$$
X_{n+j}=\partial_{y_{j}}-2 x_{j} \partial_{t}, \quad j=1, \ldots, n
$$

and the vertical space $V_{2}$ is spanned by $\partial_{t}$. In fact, $\mathbb{H}^{n}$ is a prototypical example of Carnot group of step 2. As is well known, the following Hormander bracket generating condition holds,

$$
\left[X_{i}, X_{n+j}\right]=-4 \delta_{i j} \partial_{t}, \quad \forall i, j \in\{1, \ldots, n\}
$$

and consequently the sub-Laplacian defined by

$$
\Delta_{\mathbb{H}} u=\sum_{i=1}^{2 n} X_{i}^{2} u
$$

is hypoelliptic. Note that in real coordinates we have,

$$
\Delta_{\mathbb{H}} u=\Delta_{z} u+\frac{|z|^{2}}{4} \partial_{t}^{2} u+\partial_{t} T u
$$

where

$$
T u=\sum_{i=1}^{n}\left(y_{j} \partial_{x_{j}} u-x_{j} \partial_{y_{j}} u\right)
$$

As mentioned in Introduction, $T u=0$ if and only if (1.6) holds and in which case $\Delta_{\mathbb{H}} u$ is given by

$$
\Delta_{\mathbb{H}} u=\Delta_{z} u+\frac{|z|^{2}}{4} \partial_{t}^{2} u
$$

Now we recall that in [21], Garofalo and Lanconelli showed that the following unique continuation results hold.

Theorem 5.1 (GL) Let $u$ be a solution to

$$
\Delta_{\mathbb{H}} u=V u
$$

such that

$$
|t T u(z, t)| \leq g(\rho(z, t))|z|^{2}|u(z, t)|
$$

for some Dini-integrable $g$ satisfying (1.3). Now corresponding to $N=2 n$ and $\beta=m=1$, if $V$ satisfies the growth condition as in (1.2), then $u$ satisfies the strong unique continuation property at 0 .

If instead $V$ satisfies the growth condition as in (1.4) for some $\delta$ small enough, then there exists $r_{0}>0$ such that if

$$
\int_{B_{r}} u^{2} \psi=O\left(\exp \left(-A r^{-\alpha}\right)\right), \quad \text { as } r \rightarrow 0^{+}
$$

for some $A, \alpha>0$, then $u \equiv 0$ in $B_{r_{0}}$.

As previously mentioned in Introduction, the proof in [21] is based on Almgren-type frequency function approach. Now in the situation of Theorem 5.1 when $T u \equiv 0$, we obtain the following improvement of Theorem 5.1 as a consequence of our unique continuation results in Theorems 4.2 and 4.3 in Sect. 4. 


\section{Theorem 5.2 Let $u$ be a solution to}

$$
\Delta_{\mathbb{H}} u=V u, \quad \text { in } B_{r_{0}}
$$

such that $u, X_{i} u, X_{i} u, X_{i} X_{j} u \in L^{2}\left(B_{r_{0}}(0,0)\right), \forall i, j \in\{1, \ldots, 2 n\}$ and $V$ satisfies either the growth condition as in (1.7) or as in (1.8) corresponding to $\beta=m=1$ and $N=2 n$. Moreover, assume that $T u \equiv 0$. Then if $u$ vanishes to infinite order at 0 in the sense of (4.3), then $u \equiv 0$ in $B_{r_{0}}$.

Proof In view of the discussion around (5.3) above, we note that

$$
\Delta_{\mathbb{H}} u=\Delta_{z} u+\frac{|z|^{2}}{4} \partial_{t}^{2} u, \quad z=(x, y) \in \mathbb{R}^{2 n}
$$

i.e. up to a normalization factor of 4 , we have that $\Delta_{\mathbb{H}} u=\mathcal{B}_{1} u$. Now if $V$ satisfies (1.7), we can apply the result in Theorem 4.2 to conclude that $u \equiv 0$.

On the other hand, if $V$ instead satisfies the growth condition in (1.8), we apply the result in Theorem 4.3 to again conclude that the desired conclusion holds. Note that in our case, we have that $N=2 n$, and hence, Theorem 4.3 can be applied which corresponds to the case when $N$ is even. We would, however, like to direct the attention of the reader to a subtle aspect in the application of Theorem 4.3. The reader should note that in the proof of Theorem 4.3, we needed at an intermediate step that $\left|\nabla_{z} u\right|^{2}+|z|^{2}\left|\partial_{t} u\right|^{2} \in L^{1+\gamma}$ for some $\gamma>0$ in order to assert by an application of Hölder and interpolation-type inequality that $(\sin \phi)^{-\epsilon}\left(\left|\nabla_{z} u\right|^{2}+|z|^{2}\left|\partial_{t} u\right|^{2}\right)$ vanishes to infinite order in the $L^{1}$ mean at the point $(0,0)$ for small enough $\epsilon$. That was also needed to ensure the finiteness of the quantity in (4.25). In our situation, this is guaranteed by the fact that since $T u=0$, therefore we have

$$
\sum_{i=1}^{2 n} X_{i}^{2} u=\left|\nabla_{z} u\right|^{2}+|z|^{2}\left(\partial_{t} u\right)^{2}
$$

and consequently by the Folland-Stein embedding ([14]), the higher integrability of $\left|\nabla_{z} u\right|^{2}+$ $|z|^{2}\left|\partial_{t} u\right|^{2}$ follows. The rest of the proof remains the same.

Acknowledgements One of us, A.B., would like to thank Prof. Nicola Garofalo for several suggestions and feedbacks related to this work.

\section{References}

1. Amrein, W., Berthier, A., Georgescu, V.: $L^{p}$ inequalities for the Laplacian and unique continuation. Ann. Inst. Fourier, Grenoble 31(3), 153-168 (1981)

2. Aronszajn, N., Krzywicki, A., Szarski, J.: A unique continuation theorem for exterior differential forms on Riemannian manifolds. Ark. Mat. 4(1962), 417-453 (1962)

3. Almgren, F.: Dirichlet's problem for multiple valued functions and the regularity of mass minimizing integral currents, minimal submanifolds and geodesics (Proc. Japan-United States Sem., Tokyo, 1977), pp. 1-6, North-Holland, Amsterdam-New York, (1979)

4. Banerjee, A.: Sharp vanishing order of solutions to stationary Schrodinger equations on Carnot groups of arbitrary step. J. Math. Anal. Appl. 465(1), 571-587 (2018)

5. Banerjee, A., Garofalo, N.: Quantitative uniqueness for zero-order perturbations of generalized BaouendiGrushin operators. Rend. Istit. Mat. Univ. Trieste 48, 189-207 (2016)

6. Baouendi, M.S.: Sur une classe d'operateurs elliptiques degeneres. (French). Bull. Soc. Math. France 95, 45-87 (1967)

7. Bahouri, H.: Non prolongement unique des solutions d'opérateurs "somme de carrés" (French) [Failure of unique continuation for "sum of squares" operators]. Ann. Inst. Fourier (Grenoble) 36, 137-155 (1986) 
8. Carleman, T.: Sur un probleme d'unicite pur les systemes d'equations aux derivees partielles a deux variables independantes. Ark. Mat. Astr. Fys. 26(17), 9 (1939)

9. Chanillo, S., Sawyer, E.: Unique continuation for $\Delta+v$ and the C. Fefferman-Phong class. Trans. Am. Math. Soc 318(1), 275-300 (1990)

10. Capogna, L., Danielli, D., Garofalo, N.: An embedding theorem and the Harnack inequality for nonlinear subelliptic equations. Commun. PDE 18, 1765-1794 (1993)

11. Caffarelli, L., Salsa, S., Silvestre, L.: Regularity estimates for the solution and the free boundary of the obstacle problem for the fractional Laplacian. Invent. Math. 171(2), 425-461 (2008)

12. Franchi, B., Gutierrez, C., Wheeden, R.: Two-weight Sobolev-Poincare inequalities and Harnack inequality for a class of degenerate elliptic operators. Atti Accad. Naz. Lincei Cl. Sci. Fis. Mat. Natur. Rend. Lincei (9) Mat. Appl. 5(2), 167-175 (1994)

13. Franchi, B., Lanconelli, E.: Hölder regularity theorem for a class of linear nonuniformly elliptic operators with measurable coefficients. Ann. Scuola Norm. Sup. Pisa Cl. Sci. (4) 10(4), 523-541 (1983)

14. Folland, G., Stein, E.: Estimates for $\overline{\partial_{b}}$ complex and analysis on the Heisenberg group. Commun. Pure Appl. Math. 27, 429-522 (1974)

15. Escauriaza, L.: Carleman inequalities and the heat operator I. Duke Math. J. 104(1), 113-127 (2000)

16. Escauriaza, L., Vega, L.: Carleman inequalities and the heat operator II. Indiana Univ. Math. J 50(3), 1149-1169 (2001)

17. Escauriaza, L., Seregin, G., Sverak, V.: Backward uniqueness for parabolic equations. Arch. Ration. Mech. Anal. 169(2), 147-157 (2003)

18. Garofalo, N.: Unique continuation for a class of elliptic operators which degJ. Differential Equationsenerate on a manifold of arbitrary codimension. J. Differ. Equ. 104(1), 117-146 (1993)

19. Garofalo, N., Lin, F.: Monotonicity properties of variational integrals, $A_{p}$ weights and unique continuation. Indiana Univ. Math. J. 35, 245-268 (1986)

20. Garofalo, N., Lin, F.: Unique continuation for elliptic operators: a geometric-variational approach. Commun. Pure Appl. Math. 40, 347-366 (1987)

21. Garofalo, N., Lanconelli, E.: Frequency functions on the Heisenberg group, the uncertainty principle and unique continuation. Ann. Inst. Fourier (Grenoble) 40, 313-356 (1990)

22. Grušin, V.V.: A certain class of hypoelliptic operators. (Russian) Mat. Sb. (N.S.) 83(125), 456-473 (1970)

23. Grušin, V.V.: A certain class of elliptic pseudodifferential operators that are degenerate on a submanifold. (Russian) Mat. Sb. (N.S.) 84(126), 163-195 (1971)

24. Garofalo, N., Rotz, Kevin: Properties of a frequency of Almgren type for harmonic functions in Carnot groups. Calc. Var. Partial Differ. Equ. 54(2), 2197-2238 (2015)

25. Garofalo, N., Shen, Z.: Carleman estimates for a subelliptic operator and unique continuation. Ann. Inst. Fourier (Grenoble) 44(1), 129-166 (1994)

26. Garofalo, N., Vassilev, D.: Strong unique continuation properties of generalized Baouendi-Grushin operators. Commun. Partial Differ. Equ. 32(4-6), 643-663 (2007)

27. Jerison, D.: Carleman inequalities for the Dirac and Laplace operators and unique continuation. Adv. Math. 62(2), 118-134 (1986)

28. Jerison, D., Kenig, C.: Unique continuation and absence of positive eigenvalues for Schrodinger operators. Ann. Math. (2) 121(3), 463-494 (1985)

29. Koch, H., Petrosyan, A., Shi, W.: Higher regularity of the free boundary in the elliptic Signorini problem. Nonlinear Anal. 126, 3-44 (2015)

30. Koch, H., Tataru, D.: Carleman estimates and unique continuation for second-order elliptic equations with nonsmooth coefficients. Commun. Pure Appl. Math. 54(3), 339-360 (2001)

31. Pan, Y.: Unique continuation for Schrodinger operators with singular potentials. Commun. Partial Differ. Equ. 17(5-6), 953-965 (1992)

32. Poon, C.C.: Unique continuation for parabolic equations. Commun. Partial Differ. Equ. 21(3-4), 521-539 (1996)

33. Xu, C.J.: Subelliptic variational problems. Bull. Soc. Math. France 118, 147-169 (1990)

Publisher's Note Springer Nature remains neutral with regard to jurisdictional claims in published maps and institutional affiliations. 